\title{
Virus as Evolutionary Product of the World of Biopolymers
}

\author{
VA Dementiev* \\ V Vernadsky Institute of Geochemistry RAS, Russia \\ *Corresponding author: VA Dementiev, V. Vernadsky Institute of Geochemistry RAS, Russia
}

\begin{tabular}{lll}
\hline ARTICLE INFO & & Abstract \\
\cline { 1 - 1 } $\begin{array}{l}\text { Received: May 06, } 2020 \\
\text { Published: }\end{array}$ & & $\begin{array}{l}\text { Citation: VA Dementiev. Virus as Evolutionary Product of the World of Biopolymers. } \\
\text { Biomed J Sci \& Tech Res 27(4)-2020. BJSTR. MS.ID.004541. }\end{array}$ \\
\hline
\end{tabular}

\section{Opinion}

There are various hypotheses about the origin of viruses in the literature. We strongly disagree with a hypothesis of the emergence of viruses as a result of the evolution of the prebiological world of complex organic molecules. As arguments, we will use the experience of computer simulation of the evolution of the simplest organic world, which includes amino acids, nucleic bases, ATP and a neutral dense medium [1]. These computer experiments took into account not only strong peptide bonds, but also hydrogen bonds and comparatively weak physical forces of interaction between complex radicals of amino acid residues [2]. Along the way, the complexity of the polypeptide chains and its change during the evolution of the model chemical world were evaluated [3]. We will also refer to one of the driving forces of the evolution of the microworld, to thermal motion in the medium and in biopolymers. This universal form of movement provides both random encounters of molecules for their evolutionary complication and for their movement towards Life, as well as the obligatory destruction of complex molecules, their Death. The latter is a necessary condition for the assembly of destruction products into new complex structures, that is, again for movement towards Life [4].

The rejected hypothesis suggests that in the Ocean, where a rather vast pool of complex organic molecules has already been accumulated, as in the model [1], very long chains of polypeptides were spontaneously assembling due to their autocatalytic properties. Under the influence of polypeptides as catalysts, structurally corresponding polynucleotide chains arose. This process led to the formation of complexes where the polypeptide chain and the parallel chain of the encoded polynucleotide are linked by weak forces of intermolecular interaction. Thermal movement broke these bonds, and as a result, the polypeptide molecule and its separately living genetic memory were released in the form of a strictly corresponding polynucleotide molecule. This made it possible to collect new identical copies of the randomly generated original polypeptide on the polynucleotide matrix. So, a primitive mechanism of multiplication of complex molecules arose. Let's look at the molecular structure of the polypeptide-polynucleotide complex. It is very similar to the structure of the virion, a separate particle of a modern virus. If you fold the structure of the complex into a ball, then on its surface there may appear an object resembling a protein, and inside the ball there may be a thread resembling DNA or RNA. What is not a virus?

However, the described structure cannot function as a virion in the prebiological organic world. Virion will inevitably face thermal death, that is, the division into two separate strands of polymers of different chemical classes. For the evolution of the chemical world, this is just what is needed, but not for the alleged viral particle. The virion should remain for some time until it fulfills its specific task to multiply with other more complex structures aid. Let us imagine a case where such strong bonds appeared between two chains of a polymer complex that at a low temperature of the medium the thermal motion is unable to disengage the two polymer molecules. This is possible if the polypeptide mainly contains amino acid residues with the most complex radicals. Then the complex object can wander randomly for a relatively long time in a neutral environment, resembling the expression of the virus. However, it was shown [5] that, in long polymer chains, the thermal vibrational energy of the environment inevitably accumulates, breaking these chains into fragments. 
The polypeptide chain is most susceptible to this transformation, while a polynucleotide chain such as DNA is more stable. This is well known in forensics. So, in the prebiological world, the likely contender for the role of the virus will inevitably be destroyed, failing to manifest its specific properties. We propose to consider a different hypothetical mechanism for the emergence and functioning of the virus in a modern animal cell with its sophisticated biochemical life. In such a biochemical reactor, acts of protein synthesis and structures such as mRNA and tRNA are repeatedly reproduced. Nuclear DNA replication necessarily takes place before cell division. Due to the complexity of protein structures, errors in their assembly from amino acids produced by the digestive system of a large animal organism are possible. Mutations in mRNA and tRNA molecules are possible as well. As a result, a complex of complementary biopolymers may arise, which are extremely strongly connected by intermolecular forces due to acid residues with very complex radicals. It turns out a stable virion that can exist independently both in the body and in the external environment. In the body, it can exist for a long time.

If thermal movement tears a fragment from the protein coat, the protein richness of the body will easily provide the virion with material to repair the damage. In the external environment, the virion has much less time. Sooner or later in the warm season, the entire protein shell of the virion will be destroyed. There will be a naked carrier of the genetic information of the virus. If it accidentally enters a certain living organism, then without the help of the protein coat he will have nothing to do there. Without the specific interaction of the virion protein with the cell receptor, it will not be able to mislead the cell that the virion protein is similar to the protein needed for the cell and that it is necessary to allow the virion into the cell. Further, the virion can host in the cell in a known manner with known consequences. Another option is also possible: the virus enters the body of the species that generated it. The cell membrane easily recognizes it. But why does a cell need such a protein, if there is enough of such a protein in the cell itself. The cell will remain indifferent to the virus. And thermal motion will eventually kill the protein shell of the virion. The disease will not take place. Thus, it can be assumed that viruses arose during the evolution of living matter against the background of a very rich composition of biopolymers in a modern cell with its diverse biochemical life. The high mutability of viruses follows from this, since in the very act of random formation of the structure of a new virion, the most important is the variability of the structure of protein molecules in the cytoplasm of the parent cell of this virion.

\section{References}

1. Dementiev VA (2018) Origin of the simplest genetic code as an evolutionary stage of the Earth. Geochemistry International 56: 65-70.

2. Dementiev VA (2018) Interaction of radicals in polypeptides. Current Research in Biopolymers 2018(Sp 1).

3. Dementiev VA (2018) Quantitative description of the course of chemical evolution. Current Research in Biopolymers 2018(2).

4. Dementiev VA (2020) How to: the origin of life or the rise of life and death? Biomedical Journal of Scientific \& Technical Research 26(1): 19627-19628.

5. Gribov LA, Dementiev VA (2010) Wave motions in molecular nanostructures: results of computer experiments. Journal of Structural Chemistry 51: 331-336.
ISSN: $2574-1241$

DOI: $10.26717 /$ BJSTR.2020.27.004541

VA Dementiev. Biomed J Sci \& Tech Res

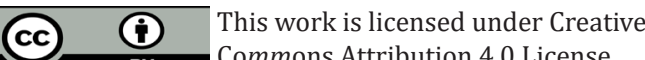

Submission Link: https://biomedres.us/submit-manuscript.php

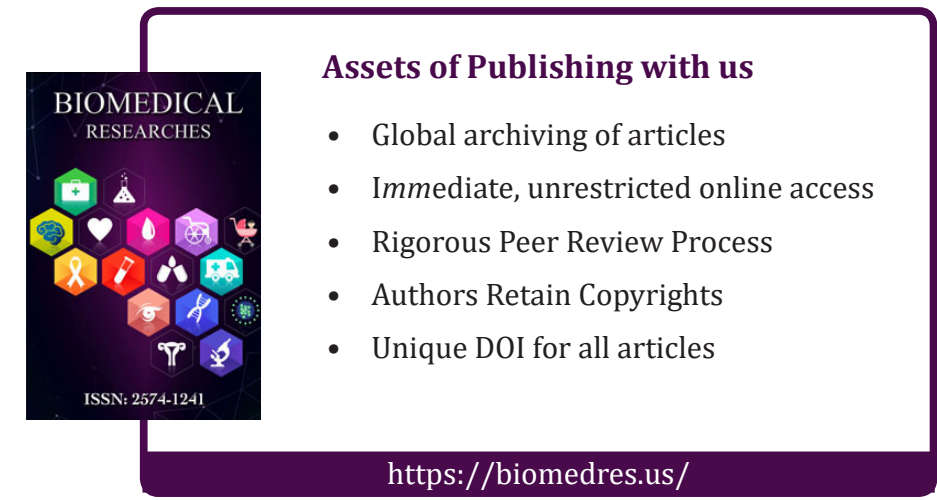

